\title{
An improved method for preparation of mouse retinal cryosections
}

\author{
Ling Li, Yongqiong Lin, Fang Yang, Tongdan Zou, Jialiang Yang, Houbin Zhang \\ The Key Laboratory for Human Disease Gene Study of Sichuan Province and Institute of Laboratory Medicine, \\ Sichuan Provincial People's Hospital, University of Electronic Science and Technology of China, Chengdu, Sichuan, \\ China
}

Immunohistochemistry using mouse retinal cryosections is a routine assay used in vision research. However, retinal tissues are fragile, and it is difficult to obtain an ideal retinal cryosection. Here, we developed a modified method for preparing retinal cryosection. Super Glue was applied on the surface of the sclera before the cornea and the lens are removed from either the unfixed or PFA-fixed mouse eyeballs. The new methods largely prevented retinal detachment in mouse retinal cryosections. Immunostaining of retinal cryosections derived from PFA-fixed mouse eyes using rod and cone markers yielded high-quality immunofluorescent images. Immunolabeling of retinal cryosections obtained from unfixed mouse eyes using a cilium-specific marker had improved orientations of photoreceptor connecting cilia. This new method substantially improves the morphology and immunostaining results of fixed and unfixed mouse eyes.

Key words: Retina; cryosections; immunocytochemistry; glue.

Correspondence: Houbin Zhang, The Sichuan Provincial Key Laboratory for Human Disease Gene Study, Sichuan Provincial People's Hospital, University of Electronic Science and Technology of China, 32 The First Ring Road West 2, Chengdu, Sichuan, 610072, China.

Tel. +86.28.87393375 - Fax: +86.28 .87393596 .

E-mail: houbin_zhang@yahoo.com

Contributions: LL, YL and FY contributed equally. All the authors made a substantive intellectual contribution, performed part of the experiments. All the authors have read and approved the final version of the manuscript and agreed to be accountable for all aspects of the work.

Conflict of interest: The authors declare that they have no competing interests, and all authors confirm accuracy.

Availability of data and materials: The datasets used and/or analyzed during the current study are available from the corresponding author on reasonable request.

Ethical Approval: The animal use protocol listed below has been reviewed and approved by the Animal Care and Use Committee of the Sichuan Provincial People's Hospital. 


\section{Introduction}

Mouse models with genetic modifications are widely used to study retinal diseases. To dissect the disease pathogenesis, it is necessary to detect the distribution of proteins within retinas by immunohistochemistry. Mouse eyeballs need to be embedded and sectioned prior to immunostaining. Epoxy resin, paraffin, and optimal cutting temperature (OCT) compoundare three common embedding materials that are used to produce plastic, paraffin, and cryosections, respectively.

Plastic sections preserve the retina histology extremely well, and have been extensively used for the morphology or electron microscopy (EM) study of the retina. However, plastic sections are inconvenient for normal immunocytochemical and immunofluorescent studies. Preparation of plastic-embedded sections involves use of glutaraldehyde and extended fixation of eyeballs, resulting in overwhelming autofluorescent signals that mask real immunosignals in immunofluorescent assays. ${ }^{1}$ Paraffin-embedded sections also preserve the histology very well, and have been adapted for histology study using hematoxylin and eosin staining. A typical protocol for paraffin-embedded retinal sections requires extended fixation and high concentration of formalin, which also gives rise to higher background autofluorescence. Moreover, immunostaining of plastic and paraffin-embedded sections usually requires additional processing, such as antigen retrieval, which produces higher background and results in lower signal-to-noiseratio. $^{2}$

Given the unique laminar organization and properties of the retinal tissue, it is very technically demanding to obtain murine retinal cryosections with ideal morphology. The murine retina consists of several anatomically distinct tissue structures, including retinal pigment epithelium (RPE), photoreceptor outer segments (OS), inner segments (IS), outer nuclear layer, outer plexiform layer (OPL), and inner nuclear layer (INL). The RPE layer loosely contacts the OS layer of photoreceptors under normal light conditions. They can easily separate from each other during tissue processing. Additionally, the connecting cilium is a thin structure that is fragile and susceptible to breakage, resulting in disruption of the OS from the IS. Thus, it is difficult to consistently obtain optimal results with good morphology from retinal cryosections, even for experienced technicians.

In this study, we use inexpensive Super Glue to spread over the surface of the sclera before the cornea and lens are removed, which minimizes distortion of eyeballs during processing. This modified protocol greatly improves the morphology of retinal cryosections and the quality of immunostaining images with very high reproducibility.

\section{Materials and Methods}

\section{Animals}

All animals were housed in the mouse facility of the Sichuan Provincial People's Hospital, China. All procedures described here were approved by the Animal Care and Use Committee of the Sichuan Provincial People's Hospital, and in accordance with the ARVO Animal Statement and the guideline of the U.S. National Institutes of Health Guide for Care and Use of Laboratory Animals.

\section{Sectioning of a PFA-fixed mouse eyeball and immunos- taining}

Wild-type C57Black/6/J mice of varied ages (including postnatal day 12,1 month, 6 months, 1 year, and 2 years) were sacrificed by cervical dislocation. At least three mice were used for each age point. Eyeballs enucleated from mice were submerged into 4\% PFA (paraformaldehyde) prepared in 1x PBS ( $\mathrm{pH} 7.4$ ) for fixation at $4^{\circ} \mathrm{C}$ for $10 \mathrm{~min}$. A small incision was made on the central cornea area and the eyeball was allowed for fixation for an additional $2 \mathrm{~h}$ at $4^{\circ} \mathrm{C}$, followed by cryoprotection in $30 \%$ sucrose solution prepared in $1 \mathrm{x}$ PBS at $4^{\circ} \mathrm{C}$ for at least $2 \mathrm{~h}$ till the eyeball settled to the bottom of the tube. The eyeball was transferred to a petri dish and the excess sucrose solution was removed by using a kimwipe. The eyeball was positioned with the eyecup facing down. A drop of 1-1.5 $\mu \mathrm{L}$ (dependent on the size of eyeballs) Super Glue (also known as "Krazy Glue" or cyanoacrylate glue or Glue 502) was applied to the surface of the sclera and spread evenly. Approximately within one to two minutes, the glue dried and became hardened. The eyecup was flipped around and the cornea was cut away under a dissecting microscope followed by removal

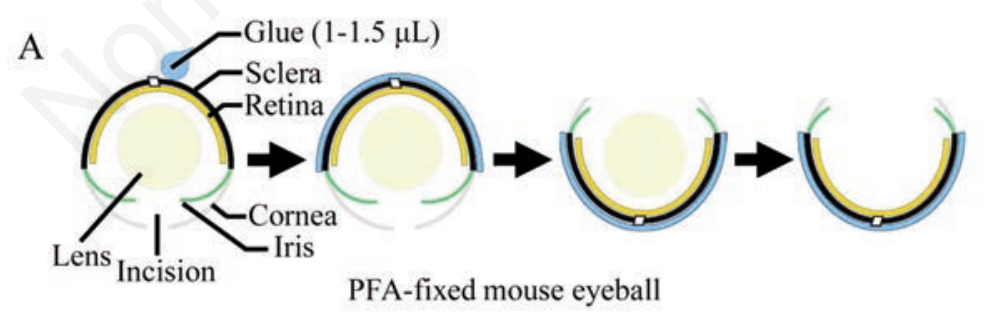

B

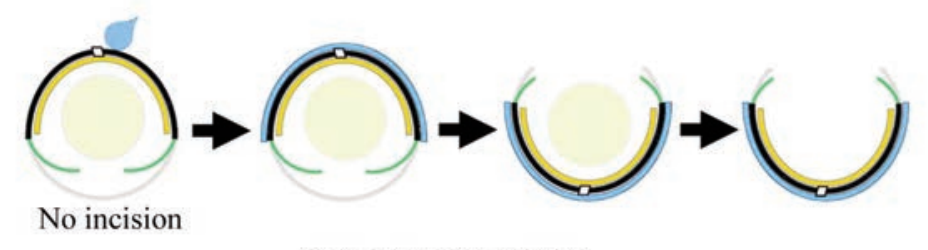

Unfixed mouse eyeball

Figure 1. Schematics showing processing of mouse eyeballs using Super Glue. A PFA-fixed (with an incision in the cornea) (A) or an unfixed fresh (without an incision in the cornea) (B) mouse eyeball is placed under a dissecting microscope with the eyecup facing down. Super Glue (1-1.5 $\mu \mathrm{L})$ is applied to the surface of the sclera and spread over the sclera. As soon as Super Glue dries (approximately 1-2 min after the glue is spread), the eyecup is flipped around followed by removal of the cornea and the lens. 
of the lens. The procedure was illustrated in Figure 1A. The eyecup was then embedded into OCT with the sagittal plane paralleling to the bottom of the embedding mold, frozen, and sectioned along the direction paralleling to the sagittal plane of the eyecup on a cryostat at $12 \mu \mathrm{m}$. For immunostaining, the cryosections were permeabilized and blocked with 5\% donkey serum diluted in $1 \mathrm{x}$ PBS containing $0.2 \%$ triton $\mathrm{X} 100$ followed by immunostaining with a rabbit polyclonal rhodopsin or S-opsin antibody (1:500, Merk Millipore, Billerica, MA, USA) as described previously. ${ }^{3}$ The dilution for the goat-anti-rabbit Alexa-488 secondary antibody (Invitrogen, Waltham, MA, USA) was 1:300. Fluorescent signals were imaged on a Zeiss confocal microscope.

\section{Sectioning of unfixed mouse eyeballs and immunostaining}

The eyeballs were enucleated and rinsed in 1x PBS briefly. The sclera was coated with a layer of Super Glue as described above (Figure 1B). The cornea and lens were removed followed by embedding of the eyecup into OCT. The tissue was sectioned on a cryostat at $12 \mu \mathrm{m}$. For immunostaining, the tissue sections were fixed with cold methanol pre-chilled at $-20^{\circ} \mathrm{C}$. The sections were immunostained with a monoclonal mouse anti-acetylated tubulin antibody (1:1,000, Sigma-Aldrich, St. Louis, MO, USA) as PFAfixed tissues.

\section{Toluidine blue staining}

For the morphology study, the retinal cryosections were stained with $0.025 \%$ Toluidine blue (Sigma-Aldrich). The sections were rinsed with $1 \times$ PBS for $2 \times 15$ min. A drop of $0.025 \%$ Toluidine blue was added to the sections and stained for $30 \mathrm{~s}$ followed by wash with water. The images were acquired by using a Zeiss light microscope.

\section{Results}

\section{Super Glue improves the morphology of cryosections of PFA-fixed mouse eyes}

According to the conventional protocol used for preparation of retinal cryosections in the eye research field, the cornea and lens need to be removed prior to embedding of the PFA-fixed eyecup into OCT. ${ }^{4}$ Even with extremely meticulous handling, this procedure may distort the eyecup and easily cause retinal detachment and disrupted photoreceptors, especially for the eyes from young mice that have softer tissues. Although fixation of the eyeballs by perfusion may improve the morphology of cryosections, it is still hard to avoid retinal detachment during removal of the cornea and lens. Furthermore, perfusion involves use of excess amount of PFA and may pose a potential threat to human health. Hence, we determined to develop an alternative approach to improving the cryosection of retinas. In view of the fact that retinal detachment and photoreceptor breakage are introduced by eyecup distortion during the process of the cornea and lens removal, and embedding, we postulated that coating of the eyecup with a hard shell on the sclera before removal of the cornea and lens can prevent eyecup distortion during tissue processing. An instant glue that dries within a minute would well serve this purpose. Here we used Super Glue (also known as "Krazy Glue" or Glue 502) that can be purchased in supermarkets. When this new method was used to prepare mouse eyecups for cryosections (see Materials and Methods), we consistently obtained desired retinal cryosections of high-quality. By using this new protocol, the retinal cryosections from onemonth-old mice showed little retinal detachment throughout the whole section (Figure 2A). We tested this new procedure on mice at different ages ranging from P12 to 2 years, and similar results were achieved for all of them (Supplementary Figure 1). Therefore, this technique can essentially be used for mouse eyes at all ages.

\section{Super Glue improves the quality of immunofluorescent images of retinal cryosections}

As shown in Figure 2B, retinal detachment and disruption of rod and cone photoreceptors were often found in immunostaining images of the cryosections produced by the traditional technique. In comparison, immunostaining of the cryosections using the new technique markedly improved the result (Figure 2C). Individual rod OS (labeled by the rhodopsin antibody) were structurally intact and discernible in the section immunostained with a rhodopsin antibody. No apparent autofluorescent signal was detected in the glue layer adjacent to the sclera layer, implicating that the glue does not produce autofluorescent signals or interfere with the real immunofluorescent signal. Immunostaining of cone photoreceptors with anti-S-opsin also yielded great results. Individual cone OS were labeled clearly by the S-opsin antibody (Figure 2C).

A

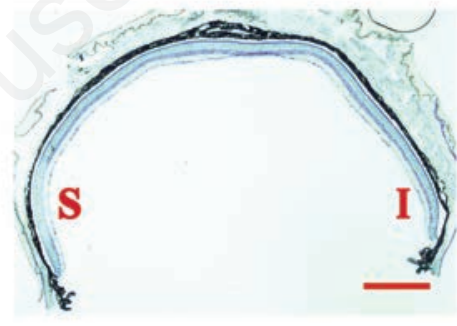

B
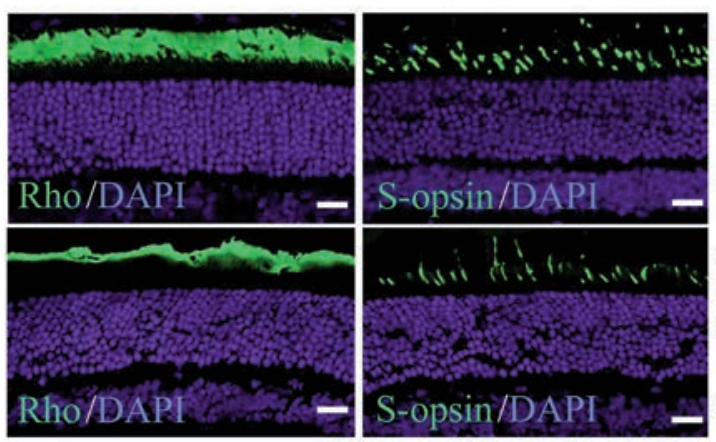

OS IS ONL OPL OS IS ONL OPL
$\mathrm{C}$

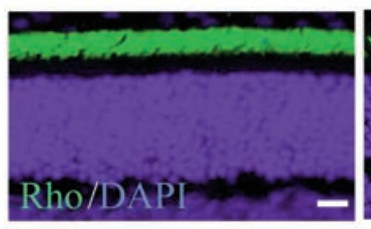

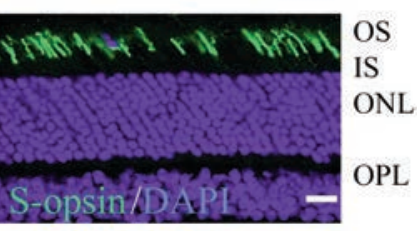

Figure 2. Staining of retinal cryosections produced from fixed mouse eyeballs by the new glue method. A) Toluidine blue staining of retinal sections from one-month old mouse $(n=5)$; $S$, superior side; I, inferior side; scale bar: $500 \mu \mathrm{m}$. B,C) Representative retinal sections derived from one-month old mice $(n=5)$ using $(B)$ or without using (C) the new technique labeled with antirhodopsin (Rho, green, left panels) or anti-S-opsin, green, right panels), and counterstained with DAPI (blue) to label nuclei; OS, outer segment; IS, inner segment; ONL, outer nuclear layer; OPL, outer plexiform layer; scale bar: $25 \mu \mathrm{m}$. 
Therefore, this glue is compatible with immunofluorescent staining. In addition to murine retinas, this new technique could be also applied to eyes from other small animals. We tested it on rat cryosections, and found that the morphology and immunostaining results of rat retinal cryosections were also dramatically improved, especially for rat eyes under one month of age (data not shown).

\section{Super Glue improves sectioning quality of unfixed reti- na tissues}

Mammalian photoreceptors are specialized ciliated cells with OS joined to IS through thin connecting cilia. The connecting cilium houses a variety of proteins required for ciliogenesis or maintenance of axoneme stability, including Cep290, ${ }^{5}$ centrins, ${ }^{6}$ RPGR, ${ }^{7}$ acetylated- $\alpha$-tubulin, ${ }^{8}$ and so on. Some of these proteins, such as RPGR, and acetylated- $\alpha$-tubulin can be immunostained only if the retinal sections are sectioned from unfixed fresh tissues. ${ }^{9}$ Traditionally, to section unfixed eyeballs, eyeballs are embedded into OCT without removal of the cornea and lens because unfixed tissues are extremely soft; otherwise, the eyecup may collapse after the lens is removed. The eyeball is intact during embedding, and no OCT is infiltrated into the eyecup. Immunolabeling of proteins within the connecting cilium for this kind of retinal cryosections usually result in a less-organized morphology (Figure 3A). By comparison, when we used the new technique described in the present study and processed the eyecups as shown in Figure 1B, the lens could be removed while maintaining the overall shape of the eyecup without distortion. By using this approach, the eyecup could be filled with OCT during embedding, which improved the immunostaining result of the connecting cilium, with better alignment and orientation (Figure 3B). The aacetylated-tubulin antibody specifically labels the ciliary axoneme in the photoreceptor connecting cilium. Therefore, this new technique can improve the orientation of the retinal cells and photoreceptor cilium in immunostaining for retinal cryosections prepared from both fixed and unfixed eyeballs.

\section{Discussion}

In this study, we use Super Glue to improve the quality and consistency of retinal cryosections. Super Glue was invented by Harry Coover, an American chemist, in 1951. The main ingredient of Super Glue is cyanoacrylate. Cyanoacrylate polymerizes into long-chain molecules in the presence of water. Therefore, when
Super Glue is spread on the wet surface of an eyeball, polymerization reaction is initiated and a relatively hard coating is formed on the surface of the eyeballs, which prevents eyeballs from deforming. This procedure mitigates retinal detachment commonly found in retinal cryosections. Because the polymerization reaction of cyanoacrylate can be very fast and the glue becomes sticky, spreading of the glue on the sclera should be completed promptly. Otherwise, the pipette tip may stick to the eyeball. Based on our experiences, using the lateral surface of the pipette tip to spread glue on the sclera could minimize the chance of the sticking. Additionally, if too much Super Glue ( $>2 \mu \mathrm{L})$ is used, excess glue may cause sticking of the eyeball to the supporting surface. If this happens, a razor blade can be used to detach the eyeball from the surface with care.

In reality, it is quite common that multiple eyeballs need to be embedded into one mold for sectioning. Each individual eyeball should be processed separately (including coating of glue on the sclera, removal of the cornea and the lens) before they are embedded together. To prevent the eyecup from drying out, eyecups that have been finished processing earlier can be re-immersed back to the sucrose solution (for fixed eyes) or PBS (for unfixed eyes) while waiting for other eyes to be processed.

From our own experiences and many other researchers', it is not easy to consistently produce retinal cryosections of high quality from mouse eyes. Poor quality of retinal sections may waste animals that have been maintained for a long time or require enormous efforts for breeding. This new procedure enables us to consistently produce retinal cryosections of high quality. Our success rate is nearly $100 \%$ for this new procedure, which is contrasted to $20-60 \%$ success rate for the traditional technique, dependent on the age of mice and operators' experiences. We tested our new procedure on fixed mouse eyeballs and fresh unfixed mouse eyeballs, respectively. We obtained far better results in both cases, compared to the traditional procedure. For young mice, it is almost impossible to remove the lens from the unfixed eyeball. Our new procedure successfully circumvents this problem. We are able to remove the lens before embedding for fresh unfixed eyeballs from mice as young as P3 (unpublished data).

The neural retina is a very soft tissue lining along the inner surface of the eyecup. The sclera is the outer layer of the eyecup and mainly made up of connective fibers. Although much harder than the retina, the sclera tends to deform during fixation and tissue processing. The Super Glue coating around the sclera is even harder than the sclera, adding additional layer of protection of the eyecup from deformation during tissue processing. Despite the difference
A

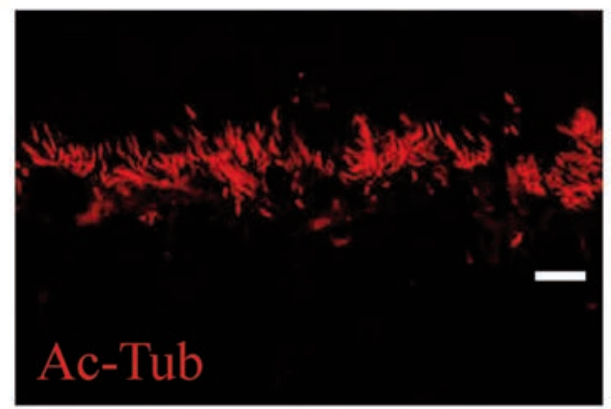

B

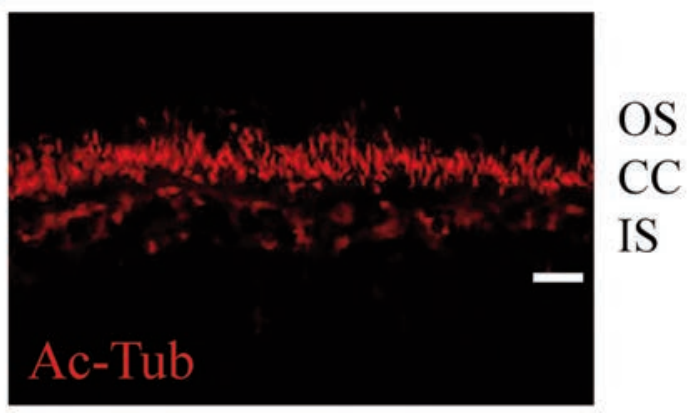

Figure 3. Immunostaining of retinal cryosections produced from fresh-embedded mouse eyeballs. Mouse retinal sections ( $\mathrm{n}=3$ ) produced with (A) or without (B) from one-month mice using Super Glue were stained by anti-acetylated $\alpha$-tubulin (Ac-Tub, red); OS, outer segment; CC, connecting cilium; IS, inner segment. Scale bar: $25 \mu \mathrm{m}$. 
in hardness of the retina, sclera, and Super Glue coating, it does not cause problems when the eyecups are sectioned on the cryostat, as indicated by the morphology of retinal sections stained with Toluidine.

In summary, due to the unique properties of the retina, it is difficult to produce cryosections of consistently high quality. The new method described in this study requires little effort to produce high-quality retinal cryosections, for both fixed and unfixed eyeballs. This new method makes the much-technique-demanding experiment easier, which will save researchers' time as well as precious animal materials.

\section{Acknowledgements}

This research project was supported by the National Precision Medicine Project (2016YFC0905200) (H.Z.), the National Natural Science Foundation of China (81570882 (H.Z.), 81770935 (H.Z.), and the grant from the Department of Science and Technology of Sichuan Province, China (2020YJ0445) (H.Z.).

\section{References}

1. Lee K, Choi S, Yang C, Wu HC, Yu J. Autofluorescence generation and elimination: a lesson from glutaraldehyde. Chem Commun (Camb) 2013;49:3028-30.

2. Chatterjee S. Artefacts in histopathology. J Oral Maxillofac
Pathol 2014;18:S111-6.

3. Zhang H, Hanke-Gogokhia C, Jiang L, Li X, Wang P, Gerstner $\mathrm{CD}$, et al. Mistrafficking of prenylated proteins causes retinitis pigmentosa 2. FASEB J 2015;29:932-42.

4. Leger H, Santana E, Beltran WA, Luca FC. Preparation of mouse retinal cryo-sections for immunohistochemistry. J Vis Exp 2019;;(149):10.3791/59683.

5. Chang B, Khanna H, Hawes N, Jimeno D, He S, Lillo C, et al. In-frame deletion in a novel centrosomal/ciliary protein CEP290/NPHP6 perturbs its interaction with RPGR and results in early-onset retinal degeneration in the rd16 mouse. Hum Mol Genet 2006;15:1847-57.

6. Ying G, Frederick JM, Baehr W. Deletion of both centrin 2 (CETN2) and CETN3 destabilizes the distal connecting cilium of mouse photoreceptors. J Biol Chem 2019;294:3957-73.

7. Hong DH, Pawlyk BS, Shang J, Sandberg MA, Berson EL, Li T. A retinitis pigmentosa GTPase regulator (RPGR)-deficient mouse model for X-linked retinitis pigmentosa (RP3). P Natl Acad Sci USA 2000;97:3649-54.

8. Arikawa K, Williams DS. Acetylated alpha-tubulin in the connecting cilium of developing rat photoreceptors. Invest Ophthalmol Vis Sci 1993;34:2145-9.

9. Zou J, Luo L, Shen Z, Chiodo VA, Ambati BK, Hauswirth WW, et al. Whirlin replacement restores the formation of the USH2 protein complex in whirlin knockout photoreceptors. Invest Ophthalmol Vis Sci 2011;52:2343-51.

Received for publication: 22 June 2020. Accepted for publication: 24 August 2020.

This work is licensed under a Creative Commons Attribution-NonCommercial 4.0 International License (CC BY-NC 4.0).

(C) Copyright: the Author(s), 2020

Licensee PAGEPress, Italy

European Journal of Histochemistry 2020; 64:3154

doi:10.4081/ejh.2020.3154 